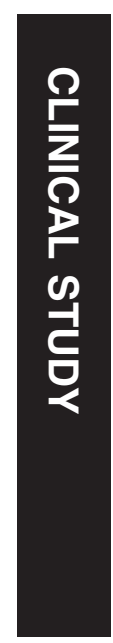

\section{Association of choroidal thickness with eye growth: a cross-sectional study of individuals between 4 and 23 years}

'Department of Ophthalmology, Izmir University Faculty of Medicine, Izmir, Turkey

${ }^{2}$ Alfagoz Eye Center, Izmir, Turkey

${ }^{3}$ Retina Eye Hospital, Bursa, Turkey

Correspondence: MO Zengin, Gursel Aksel Bulvarı, No. 14, 35350, Uckuyular, Izmir, Turkey Tel: +90 232 2464949; Fax: +90232 2240909 . E-mail: mehmetozgurzengin@ yahoo.com

Received: 4 June 2014 Accepted in revised form: 21 August 2014 Published online: 3 October 2014

This work has not been presented in any meeting. There was no proprietary interest or funding provided for this study.

\begin{abstract}
Background and aims We aimed to evaluate the association of subfoveal choroidal thickness (SFCT) with age and to determine its relationship with axial length (AL) and ocular biometric parameters, in children and young adults during growth period. Methods One hundred and sixty patients ( 80 male, 80 female) aged between 4 and 23 years were included. Patients were classified into five groups according to their ages as group 1 (4-7 years of age), group 2 (8-11 years), group 3 (12-15 years), group 4 (16-19 years), and group 5 (20-23 years). SFCT was assessed using spectral-domain optical coherence tomography (3D OCT-2000). The measurements were taken at the same daytime (1000-1200 hours) to avoid diurnal fluctuation. Ocular AL and anterior segment parameters were measured using optical biometry (Lenstar LS900)).

Results The average SFCT was $308.1 \pm 47.6 \mu \mathrm{m}$, ranging from 206 to $410 \mu \mathrm{m}$. The mean SFCT values in group 1 to 5 were measured as $306.8 \pm 42.0,297.8 \pm 48.1$, $283.2 \pm 38.9,326.9 \pm 57.4$, and $325.8 \pm 35.9 \mu \mathrm{m}$, respectively. The mean CT of group 3 was significantly thinner than group 4 and 5 ( $P<0.05$ for two groups), however, there was no statistical significance compared with group $1(P=0.227)$ and group $2(P=0.693)$. On stepwise regression analysis, age exhibited a positive association with SFCT ( $B=2.8, P<0.001)$ and AL exhibited a negative association with choroidal thickness $(B=-16.7, P<0.001)$.

Conclusions We found that SFCT shows no age-related difference until age of 15 , whereas 16-23-year old participants had
\end{abstract}

MO Zengin ${ }^{1}$, E Karahan² , S Yilmaz ${ }^{3}$, E Cinar ${ }^{1}$, I Tuncer ${ }^{2}$ and C Kucukerdonmez ${ }^{1}$ thicker SFCT compared with the younger ones. In that aspect, a thicker choroid associated with age in certain age groups suggests an age period of SFCT increase to a plateau in young adulthood. Larger studies are warranted to elucidate the age-related changes in SFCT in childhood, as improved evaluation of normal choroidal thicknesses during eye growth should assist in the diagnosis of choroidal abnormalities associated with eye disease.

Eye (2014) 28, 1482-1487; doi:10.1038/eye.2014.227; published online 3 October 2014

Introduction

The choroid, one of the most highly vascularized tissues of the body, has important roles in delivery of oxygen and nutrients to the outer retina, modulation of retinal temperature, adjustment of retinal position, and secretion of growth factors. ${ }^{1}$ A technique known as enhanced depth imaging optical coherence tomography (EDI-OCT) enables in vivo crosssectional imaging of the choroid. ${ }^{2}$ Several recent studies using commercially available and custom-developed Fourier-domain EDI-OCT have afforded new insights into the in vivo structural characteristics of the normal adult human choroid. ${ }^{3-6}$ The average choroidal thickness in the subfoveal region of healthy adults (over 18 years of age) is $192-354 \mu \mathrm{m}$. Thickening of the choroid may be mechanistically linked to the synthesis of scleral macromolecules, and thus may have important roles in the homeostatic control of eye growth and the etiologies of myopia and hyperopia. ${ }^{1}$ However, although our understanding of 
choroidal characteristics has increased substantially in recent years, only a few reports to date have provided values for choroidal thickness in children. ${ }^{7-9}$ In a recent study, age and axial length (AL) were determined to be negatively correlated with choroidal thickness and volume in adults. ${ }^{7}$ Similarly, in a previous study, we also demonstrated a decrease in choroidal thickness with increasing myopia, age, and AL in adults. ${ }^{8}$ However, although our understanding of choroidal characteristics has increased substantially in recent years, only a few reports to date have provided values for choroidal thickness in children. ${ }^{9-11}$ Recently, an increase in choroidal thickness with age but an inverse correlation of choroidal thickness with AL was also reported in healthy children aged $3.5-14.9$ years. ${ }^{11}$

Improved evaluation of normal choroidal thicknesses during eye growth should assist in the diagnosis of choroidal abnormalities associated with eye disease, and also allow us to understand the differences in the choroid during normal eye growth and the development of refractive error. Thus, we aimed to evaluate the association of subfoveal choroidal thickness (SFCT) with age and to determine its relationship with AL and ocular biometric parameters during growth period, in children and young adults.

\section{Materials and methods}

This study involved 160 eyes of 160 healthy Turkish people aged between 4 and 23 years, who visited the ophthalmology clinic for routine examinations. The study protocol was approved by Izmir University Institutional Review Board and Ethics Committee (TAEK-010-2013). The research adhered to the tenets of the Declaration of Helsinki, and all participating children and their parents provided a detailed written informed consent.

Patients were classified into five groups (each included 32 patients) according to their ages: 4-7 years (group 1), 8-11 years (group 2), 12-15 years (group 3), 16-19 years (group 4), and 20-23 years (group 5). All groups were matched $1: 1$ for gender. According to this, group 1 and 2 were classified as children, group 3 adolescence, and group 4 and 5 as young adults.

Only children aged 4 years or older were included because younger children were considered to be unable to cooperate with OCT examination. Patients were excluded if they had a history of ocular surface disorder, previous ocular surgery, ocular injury or history of any ocular abnormalities including strabismus, amblyopia, congenital cataract, and vitreoretinal disorders, or if they had a history of any chronic drug use including the analgesics, decongestants, and antihistamines and had any history of systemic disease, hypertension, and smoking. Moreover, children with a history of prematurity or low birth weight were also excluded. Patients who had a spherical equivalent refraction greater than -2.0 or +2.0 diopters were excluded from the study. Only right eyes of each child were evaluated. All participants underwent a detailed ophthalmic examination, including visual acuity testing, cycloplegic refraction, and evaluation of ocular alignment status, biomicroscopy, fundus examination, and choroidal thickness measurements by OCT. The spherical equivalent was calculated as the sphere plus half a cylinder. Ocular AL and anterior segment parameters were measured using optical biometry (Lenstar LS900; Haag-Streit AG, Koeniz, Switzerland).

Choroidal thickness measurements were performed by the same experienced technician using a spectral-domain OCT device ( $\lambda=840 \mathrm{~nm}, 27000 \mathrm{~A}$-scans $/ \mathrm{s}$, and $5 \mu \mathrm{m}$ axial resolution), 3D OCT-2000 (Topcon; Topcon Corp., Tokyo, Japan). The protocol of the enhanced choroidal mode cross scan was performed centering on the fovea. This protocol consisted of 6-mm cross lines with 1024 106Ascans/B-scans and overlapping 4 B-scans per image and direct B-scan observation was available. After the B-scan scale was adjusted to $1: 1 \mu \mathrm{m}$ and approximately doubling the size of imaging, the observer determined SFCT perpendicular from the outer edge of the hyperreflective RPE to the inner sclera, centered on the fovea using a 'caliper function', that is, a built-in linear measuring tool (Figure 1). Scans with low quality and indistinguishable choroidal-scleral border were not included and repeated. Choroidal thickness was measured perpendicularly from the outer edge of the retinal pigment epithelium to the choroid-sclera boundary at the subfoveal region. All OCT scans were performed at the same time of the day, in the morning (between 1000 and 1200 hours), to avoid diurnal fluctuations. Choroidal thickness measurements were obtained by two masked physicians (MOZ and CK). The average of the two measurements was taken; the differences between readings of the masked physicians were found to be within $10 \mu \mathrm{m}$ of the mean. If the measurements did not agree within $10 \mu \mathrm{m}$, then they were repeated, and if the inconsistency persisted, a third masked reader (EC) took a measurement. The interexaminer reproducibility of the choroidal thickness measurements was assessed by measuring the intraclass correlation coefficient (ICC).

The statistical analysis was performed with SPSS for Windows 17.0 (SPSS Inc., Chicago, IL, USA). The Levene's variance test for homogeneity was used to determine the linearity and the $P$-value was determined as insignificant $(P>0.05)$ in that test, showing that the variances of groups were equal. ANOVA was performed to group examine. Post hoc Tukey tests were used to 


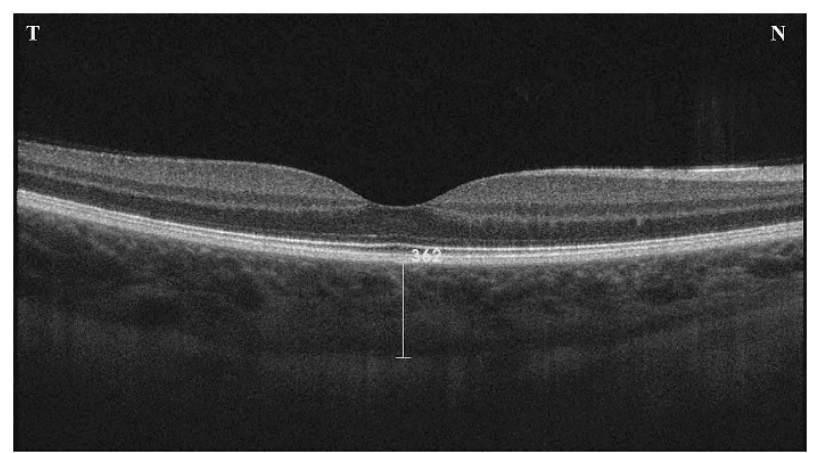

Figure 1 Example of SFCT measurements. ' $\mathrm{N}$ ' and ' $\mathrm{T}$ ' denote placements for the nasal (left) and temporal (right) quadrants.

explore between-group differences. Finally, stepwise multiple regression analysis was used to examine the influence of demographic (age and sex) and biometric (AL, central corneal thickness (CCT), anterior chamber depth (ACD), and lens thickness (LT)) factors on SFCT. $P$-value $<0.05$ was considered as statistically significant.

\section{Results}

Data analysis included 160 healthy subjects of whom 80 were male and 80 were female. The mean age of the subjects was 13.7 years (range, 4-23 years). The mean choroidal thickness for the entire population of 160 patients was $308.1 \pm 47.6 \mu \mathrm{m}$ (range, 206-410) with no statistically significant difference between male $(308.1 \pm 49.2 \mu \mathrm{m})$ and female $(308.2 \pm 46.1 \mu \mathrm{m})$ subjects $(P=0.988)$. The mean choroidal thickness values among the groups were shown in Table 1.

The mean choroidal thickness of the group 3 was the thinnest among all groups. ANOVA revealed a significant influence of age on the mean choroidal thickness $(P<0.001)$. The differences between group 3 and 4 , and group 3 and 5 on post hoc Tukey testing were statistically significant $(P 1=0.001, P 2=0.002$, respectively). However, there was no statistical significance when group 3 was compared with group 1 $(P=0.22)$ and group $2(P=0.693$; Figure 2$)$. Also, ANOVA revealed a significant influence of age on the mean choroidal thickness $(P<0.001)$. Univariate linear regression analysis showed a significant positive association between SFCT and age (slope $=6.03, r=0.19$, $P=0.015)$.

Table 1 also illustrates the mean values of ocular biometric measurements (CCT, ACD, LT, and $\mathrm{AL}$ ) for the five different age groups examined. ANOVA showed a significant influence of age for all of the biometric variables examined $(P=0.003)$. Significantly, thicker $\mathrm{CCT}$, shallower ACD, thicker crystalline lenses, and shorter AL were observed in the group 1 compared with the older age groups (Table 2). Significant difference between males and females was not observed for all of the biometric variables $(P>0.05)$.

Stepwise multiple regression analysis to examine biometric (CCT, ACD, LT, and AL) and demographic factors (age and sex) associated with SFCT revealed that age and AL were associated significantly with choroidal thickness (Table 3). The final regression model, including these predictors, was statistically significant $(P=0.003)$, with a $R^{2}$ of 0.121 . Age exhibited a positive association with SFCT $(B=2.8, P<0.001)$ and AL exhibited a negative association with choroidal thickness $(B=-16.7$, $P<0.001)$. None of the other considered predictor variables contributed significantly to the variation observed in choroidal thickness. The interexaminer ICC for the SFCT was 0.918 (95\% CI, 0.902-0.961).

\section{Discussion}

Changes in eye size during early life or childhood are in line with parameters for overall growth and physical development, ${ }^{12,13}$ and the age at cessation of growth in AL is about the same as the age at which final body height is attained. ${ }^{12}$ Such longitudinal parallelisms may suggest that eye and body size share common developmental pathways in children. Concomitant AL and body height changes with increasing age have been reported during physical development. ${ }^{14}$

In the current study, we investigated the SFCT variations during growth period of eye and body size using spectraldomain OCT. Choroidal thickness has been measured in the various populations of healthy subjects. ${ }^{4-6}$ Many recent studies have reported ocular disorders associated with altered abnormal choroidal thickness. In high myopia, retinal dystrophy, and age-related choroidal atrophy, the choroid was reported to have decreased thickness, whereas increased choroidal thickness was reported in central serous chorioretinopathy and Vogt-Koyanagi-Harada disease. ${ }^{15-19}$ Also, there is recent data on the hemodynamic effect of chemicals-sildenafil and nicotine- on choroid, obtained by OCT. ${ }^{20,21}$

In our population of 160 patients aged 4-23 years, we found a mean SFCT of $308 \mu \mathrm{m}$ with substantial variation between individuals, which was consistent with previous studies in adults. ${ }^{4,22}$ Recent studies examining in vivo choroidal thickness with OCT in children have reported mean SFCT values of $348 \mu \mathrm{m}$ (in 48 Korean children aged $4-10$ years), ${ }^{9} 330 \mu \mathrm{m}$ (in 194 children aged $4-12$ years), ${ }^{10}$ and $342 \mu \mathrm{m}$ (in 174 children aged $3.5-15$ years). ${ }^{11}$ Among these studies, only Park et al reported a negative correlation between SFCT and age. ${ }^{9}$ However, Read et $a l^{10}$ and Bidaut-Garnier $e t$ al ${ }^{11}$ have reported that choroidal thickness increases significantly from early childhood to adolescence and is inversely correlated to AL in children. It is also important to emphasize that in 
Table 1 General characteristics and mean ocular parameters of groups

\begin{tabular}{lcccccc}
\hline & $\begin{array}{c}\text { Group } 1 \\
(4-7 \text { years })\end{array}$ & $\begin{array}{c}\text { Group } 2 \\
(8-11 \text { years })\end{array}$ & $\begin{array}{c}\text { Group } 3 \\
(12-15 \text { years })\end{array}$ & $\begin{array}{c}\text { Group } 4 \\
(16-19 \text { years })\end{array}$ & $\begin{array}{c}\text { Group 5 } \\
(20-23 \text { years })\end{array}$ & $\begin{array}{c}\text { ANOVA } \\
\text { P-value }\end{array}$ \\
\hline Age & $5.90 \pm 1.02$ & $9.37 \pm 1.15$ & $13.46 \pm 0.94$ & $17.81 \pm 1.33$ & $22.21 \pm 1.10$ & 0.001 \\
Gender (F/M) & $16 / 16$ & $16 / 16$ & $16 / 16$ & $16 / 16$ & $16 / 16$ & 1.0 \\
SFCT & $306.8 \pm 42.0$ & $297.8 \pm 48.1$ & $283.2 \pm 38.9$ & $326.9 \pm 57.4$ & $325.8 \pm 35.9$ & 0.001 \\
CCT & $0.56 \pm 0.03^{\mathrm{a}}$ & $0.55 \pm 0.03$ & $0.54 \pm 0.03$ & $0.53 \pm 0.03$ & $0.53 \pm 0.03$ & 0.024 \\
ACD & $2.89 \pm 0.20^{\mathrm{b}}$ & $3.13 \pm 0.27$ & $3.22 \pm 0.20$ & $3.22 \pm 0.23$ & $3.14 \pm 0.31$ & 0.001 \\
LT & $3.65 \pm 0.13^{\mathrm{c}}$ & $3.51 \pm 0.17$ & $3.43 \pm 0.14$ & $3.46 \pm 0.2$ & $3.58 \pm 0.22$ & 0.001 \\
AL & $22.33 \pm 0.70^{\mathrm{d}}$ & $23.02 \pm 0.91$ & $23.90 \pm 0.78$ & $24.12 \pm 1.05$ & $23.79 \pm 1.12$ & 0.001 \\
\hline
\end{tabular}

Abbreviations: $\mathrm{ACD}$, anterior chamber depth; $\mathrm{AL}$, axial length; $\mathrm{CCT}$, central corneal thickness; $\mathrm{LT}$, lens thickness; $\mathrm{SFCT}$, subfoveal choroidal thickness

a The thickest central corneal thickness in group $1(P<0.05)$.

${ }^{\mathrm{b}}$ The deepest anterior chamber depth in group $1(P<0.05)$.

${ }^{\mathrm{c}}$ The thickest lens thickness in group $1(P<0.05)$.

${ }^{\mathrm{d}}$ The shortest axial length in group $1(P<0.05)$.

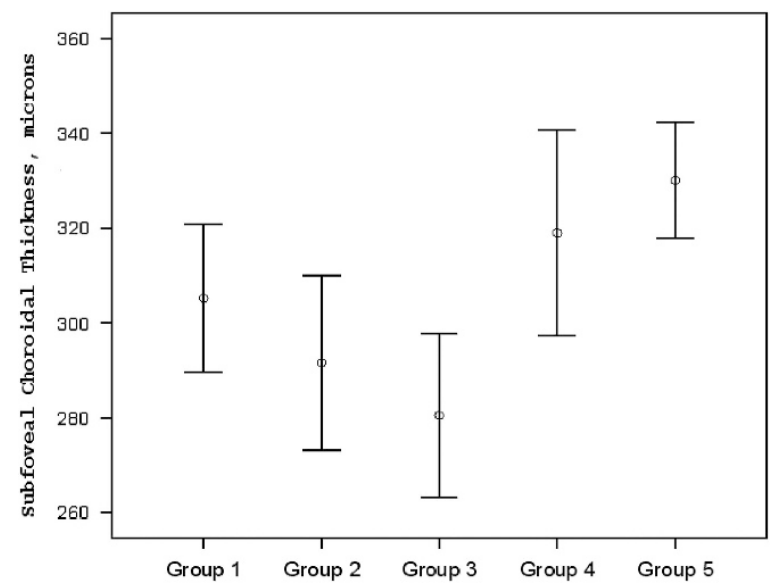

Figure 2 The average SFCT in different groups (group 1: 4-7year-old, group 2: 8-11-year old, group 3: 12-15-year-old, group 4: 16-19-year-old, and group 5: 20-23-year-old age groups).

Table 2 The $P$-values of comparisons of different groups

\begin{tabular}{llccccccccc}
\hline & $P^{1}$ & $P^{2}$ & $P^{3}$ & $P^{4}$ & $P^{5}$ & $P^{6}$ & $P^{7}$ & $P^{8}$ & $P^{9}$ & $P^{10}$ \\
\hline SFCT & 0.93 & 0.22 & 0.39 & 0.44 & 0.69 & 0.08 & 0.1 & 0.01 & 0.02 & 1.0 \\
CCT & 0.73 & 0.53 & 0.02 & 0.07 & 0.99 & 0.32 & 0.67 & 0.57 & 0.80 & 0.99 \\
ACD & 0.001 & 0.001 & 0.001 & 0.001 & 0.67 & 0.60 & 1.0 & 1.0 & 0.76 & 0.69 \\
LT & 0.01 & 0.001 & 0.001 & 0.54 & 0.48 & 0.86 & 0.45 & 0.96 & 0.01 & 0.065 \\
AL & 0.028 & 0.001 & 0.001 & 0.001 & 0.001 & 0.002 & 0.01 & 0.87 & 0.98 & 0.60 \\
\hline
\end{tabular}

Abbreviations: $\mathrm{ACD}$, anterior chamber depth; $\mathrm{AL}$, axial length; $\mathrm{CCT}$, central corneal thickness; LT, lens thickness; SFCT, subfoveal choroidal thickness.

$P^{1}$, the $P$-value of comparison of groups 1 and $2 ; P^{2}$, the $P$-value of comparison of groups 1 and $3 ; P^{3}$, the $P$-value of comparison of groups 1 and $4 ; P^{4}$, the $P$-value of comparison of groups 1 and $5 ; P^{5}$, the $P$-value of comparison of groups 2 and $3 ; P^{6}$, the $P$-value of comparison of groups 2 and $4 ; P^{7}$, the $P$-value of comparison of groups 2 and $5 ; P^{8}$, the $P$-value of comparison of groups 3 and $4 ; P^{9}$, the $P$-value of comparison of groups 3 and $5 ; P^{10}$, the $P$-value of comparison of groups 4 and 5 .

Park et $a l^{9}$ study, patients with SE $+/-6$ diopter were recruited, which was much higher SE refractive error compared with the inclusion criteria of other studies. In addition, they did not adjust AL, which is known to have
Table 3 Results of logistic regression analysis (dependent variable: subfoveolar choroid thickness)

\begin{tabular}{lrr}
\hline & \multicolumn{1}{c}{ Beta } & P-value \\
\hline Age & 0.009 & 0.999 \\
Gender & 2.795 & 0.001 \\
CCT & 0.047 & 0.668 \\
ACD & 24.046 & 0.209 \\
LT & -15.613 & 0.471 \\
AL & -16.748 & 0.001 \\
\hline
\end{tabular}

Abbreviations: $\mathrm{ACD}$, anterior chamber depth; $\mathrm{AL}$, axial length; $\mathrm{CCT}$, central corneal thickness; LT, lens thickness.

a strong correlation with $\mathrm{CT}$, in interpreting correlation between age and choroidal thickness. The differences between studies can be due to recruitment criteria, analysis, and racial differences.

Although we found a relatively thinner mean choroidal thickness compared with previous studies, the wide age range of our study population might be responsible for this difference. However, this also provides a more detailed examination of association of choroidal thickness with age. The findings of the current study revealed no age-related SFCT difference until age of 15, whereas 16-23-year old participants had thicker SFCT compared with the younger ones. Between age 16 and 23 , there seem to be no age-related difference, suggesting a plateau in SFCT development.

There are some recent studies about the effect of age on choroidal thickness. Mapelli et al ${ }^{23}$ studied 52 children with mean age of 9 years (range, 2-17 years) and reported that total and foveal choroid volume (CV) showed significant negative correlation with AL, and significant positive correlation with age. They also determined that CV increases by $0.214 \mathrm{~mm}^{3}$ (2.5\%) for every year, without grouping the children according to their ages. In a population-based cohort of 1323 healthy 11- and 12-year-old children, mean SFCT was determined as $369 \pm 81 \mu \mathrm{m}$ in girls and $348 \pm 72 \mu \mathrm{m}$ in boys. ${ }^{24}$ In that study, similar with our results, longer AL was associated 
with a thinner SFCT and there was no difference in choroidal thickness between sexes. Interestingly, in girls, the choroid was determined as the thickest in participants in the more advanced stage of pubertal development and reported to be increased with body height. The authors suggested that puberty promotes choroidal thickening in girls. Although we did not investigate the pubertal development of participants in our study, most of the cases in groups 4 and 5 (older than 15 years of age) can be considered in post-pubertal period and the mean SFCT was thicker in those groups compared with the prepubertal groups.

The exact mechanism underlying changes in choroidal thickness in early childhood remains unclear. However, these changes cannot involve passive choroidal stretching as the eye grows, as this would thin the choroid. Moreover, choroidal thickening may reflect normal growth of vascular and connective tissue, or, alternatively, changes in blood flow with age during adolescence. Changes in ocular blood flow with advancing age have been documented in adults. ${ }^{17,25}$ However, to the best of our knowledge, ocular blood flow changes during eye growth in children have not yet been examined. In marmosets and macaques, the thickness of the choroid was determined to increase by $\sim 28 \%$ from birth to puberty, when the eye is nearly adult size. ${ }^{26,27}$ Troilo et $a l^{28}$ hypothesized that choroidal thickening observed during normal development of the primate eye may slow eye growth during development, either by acting as a barrier to the diffusion of growth factors or by acting as a mechanical buffer limiting axial elongation. Thus, it is conceivable that choroidal thickening observed in our population of adolescents could also slow eye growth. Recent studies have measured in vivo choroidal thicknesses in adults; the average subfoveal thickness ranges from 192 to $354 \mu \mathrm{m}$ in diverse populations. In a recent study of young Danish adults aged 20-29 years, the average SFCT was $344 \mu \mathrm{m} .^{6}$ Thus, our finding of a mean choroidal thickness of $327 \mu \mathrm{m}$ in those aged 16-19 years suggests that choroidal thickness approaches the adult value at this age. Several cross-sectional studies in adults have clearly shown that choroidal thickness later decreases with advancing age; the mean decrease is 15 $20 \mu \mathrm{m}$ per decade in later life..$^{3,4,29}$ Our findings suggest that SFCT may increase after the age of 15 years and reaches a plateau at the age between 16 and 23 years.

There are several shortcomings regarding our study. One limitation of this study is the lack of data before 4 years of age for evident technical reasons of OCT feasibility in very young children. It would be interesting to document the CT immediately after birth to see if such a trend in choroid thickening starts earlier. In addition, longitudinal follow-up will also contribute information on CT development in children. In that aspect, Moreno et $\mathrm{al}^{30}$ reported the SFCT, with a portable SD-OCT system, in young preterm infants, term-aged preterm infants, term infants, and adults as $176 \pm 53,289 \pm 92,329 \pm 66$, and $258 \pm 66 \mu \mathrm{m}$, respectively, which were all statistically significantly different from one another, except termaged preterms to adults. In that study, the authors concluded that in preterm infants, choroidal thickness increased with age but was thinner when compared with term infants, suggesting delayed development due to retinopathy of prematurity.

Other limitation is the effect of diurnal rhythm of choroidal thickness (a change of $\sim 20-30 \mu \mathrm{m}$ ), which was demonstrated in previous studies. ${ }^{31,32}$ The OCT examinations in our study were performed at the same time of the day (1000-1200 hours), or that it may be unlikely to be a potential bias in our study. The last major limitation of this study is the fact that the choroidal thickness measurements had to be performed manually, which remains a potential cause for interobserver bias. To partly overcome this limitation, we have measured the thickness of the choroid via manual segmentation by two independent operators. The ICCs between the two operators were strong (ICC >0.90).

Despite these shortcomings, to the best of our knowledge, this is the first study about the choroidal thickness profiles during growth period from the early childhood to adult age (4-23 years) using spectral-domain OCT. We found that SFCT shows no age-related difference until age of 15, whereas 16-23-year-old participants had thicker SFCT compared with the younger ones and a thicker choroid associated with age in certain age groups, suggesting an age period of SFCT increase to a plateau in young adulthood. Larger studies are warranted to elucidate the age-related changes in SFCT in childhood, as improved evaluation of normal choroidal thicknesses during eye growth should assist in the diagnosis of choroidal abnormalities associated with eye disease.

\section{Summary}

What was known before

- The characteristics of the in vivo choroid has increased substantially in recent years, however, only a few studies have examined the thickness of the choroid in children who reported controversial results.

What this study adds

- In our study, we evaluated the choroidal thickness in children, adolescents, and young people during the growth period. According to our results were observed that choroidal thickness decreases from early childhood to adolescence, then starts to increase and reaches a peak between 16 and 19 years of age. After that, a plateau in choroidal thickness change was observed in young adults. This change in choroidal thickness during the growth period will give valuable data for future studies about this topic. 


\section{Conflict of interest}

The authors declare no conflict of interest.

\section{References}

1 Nickla DL, Wallman J. The multifunctional choroid. Prog Retin Eye Res 2010; 29: 144-168.

2 Spaide RF, Koizumi H, Pozzoni MC. Enhanced depth imaging spectral-domain optical coherence tomography. Am J Ophthalmol 2008; 146: 496-500.

3 Margolis R, Spaide RF. A pilot study of enhanced depth imaging optical coherence tomography of the choroid in normal eyes. Am J Ophthalmol 2009; 147: 811-815.

4 Ikuno Y, Kawaguchi K, Nouchi T, Yasuno Y. Choroidal thickness in healthy Japanese subjects. Invest Ophthalmol Vis Sci 2010; 51: 2173-2176.

5 Manjunath V, Taha M, Fujimoto JG, Duker JS. Choroidal thickness in normal eyes measured using Cirrus HD optical coherence tomography. Am J Ophthalmol 2010; 150: 325-329.

6 Li XQ, Larsen M, Munch IC. Subfoveal choroidal thickness in relation to sex and axial length in 93 Danish university students. Invest Ophthalmol Vis Sci 2011; 52: 8438-8441.

7 Michalewski J, Michalewska Z, Nawrocka Z, Bednarski M, Nawrocki J. Correlation of choroidal thickness and volume measurements with axial length and age using swept source optical coherence tomography and optical low-coherence reflectometry. Biomed Res Int 2014; 2014: 639160.

8 Tuncer I, Karahan E, Zengin MO, Atalay E, Polat N. Choroidal thickness in relation to sex, age, refractive error, and axial length in healthy Turkish subjects. Int Ophthalmol 2014; e-pub ahead of print 21 June 2014; doi:10.1007/s10792-0149962-4.

9 Park KA, Oh SY. Choroidal thickness in healthy children. Retina 2013; 33: 1971-1976.

10 Read SA, Collins MJ, Vincent SJ, Alonso-Caneiro D. Choroidal thickness in childhood. Invest Ophthalmol Vis Sci 2013; 54: 3586-3593.

11 Bidaut-Garnier M, Schwartz C, Puyraveau M, Montard M, Delbosc B, Saleh M. Choroidal thickness measurement in children using optical coherence tomography. Retina 2014; 34: 768-774.

12 Goss DA, Cox VD, Herrin-Lawson GA, Nielsen ED, Dolton WA. Refractive error, axial length, and height as a function of age in young myopes. Optom Vis Sci 1990; 67: 332-338.

13 Larsen JS. The sagittal growth of the eye. IV. Ultrasonic measurement of the axial length of the eye from birth to puberty. Acta Ophthalmol (Copenh) 1971; 49: 873-886.

14 Wang D, Ding X, Liu B, Zhang J, He M. Longitudinal changes of axial length and height are associated and concomitant in children. Invest Ophthalmol Vis Sci 2011; 52: 7949-7953.

15 Fujiwara T, Imamura Y, Margolis R, Slakter JS, Spaide RF. Enhanced depth imaging optical coherence tomography of the choroid in highly myopic eyes. Am J Ophthalmol 2009; 148: 445-450.

16 Yeoh J, Rahman W, Chen F et al. Choroidal imaging in inherited retinal disease using the technique of enhanced depth imaging optical coherence tomography. Graefes Arch Clin Exp Ophthalmol 2010; 248: 1719-1728.
17 Spaide RF. Age-related choroidal atrophy. Am J Ophthalmol 2009; 147: 801-810.

18 Maruko I, Iida T, Sugano Y, Ojima A, Ogasawara M, Spaide RF. Subfoveal choroidal thickness after treatment of central serous chorioretinopathy. Ophthalmology 2010; 117: 1792-1799.

19 Maruko I, Iida T, Sugano Y, Oyamada H, Sekiryu T, Fujiwara T. Subfoveal choroidal thickness after treatment of Vogt- Koyanagi-Harada disease. Retina 2011; 31: 510-517.

20 Zengin MO, Cinar E, Kucukerdonmez C. The effect of nicotine on choroidal thickness. Br J Ophthalmol 2014; 98: 233-237.

21 Kim DY, Silverman RH, Chan RV, Khanifar AA, Rondeau M, Lloyd $\mathrm{H}$ et al. Measurement of choroidal perfusion and thickness following systemic sildenafil. Acta Ophthalmol 2013; 91: 183-188.

22 Ouyang Y, Heussen FM, Mokwa N, Walsh AC, Durbin MK, Keane PA et al. Spatial distribution of posterior pole choroidal thickness by spectral domain optical coherence tomography. Invest Ophthalmol Vis Sci 2011; 52: 7019-7026.

23 Mapelli C, Dell'Arti L, Barteselli G, Osnaghi S, Tabacchi E, Clerici $\mathrm{M}$ et al. Choroidal volume variations during childhood. Invest Ophthalmol Vis Sci 2013; 54: 6841-6845.

24 Li XQ, Jeppesen P, Larsen M, Munch IC. Subfoveal choroidal thickness in 1323 children aged 11 to 12 years and association with puberty: the Copenhagen Child Cohort 2000 Eye Study. Invest Ophthalmol Vis Sci 2014; 55: 550-555.

25 Ravalico G, Toffoli G, Pastori G, Croc̀e M, Calderini S. Age related ocular blood flow changes. Invest Ophthalmol Vis Sci 1996; 37: 2645-2650.

26 Troilo D, Nickla DL, Wildsoet CF, Rada JA. Late-onset form deprivation in the marmoset produces axial myopia and reduced scleral proteoglycan synthesis [ARVO Abstract]. Invest Ophthalmol Vis Sci 1999; 40: S963.

27 Hung L-F, Wallman J, Smith III, EL. Vision dependent changes in the choroidal thickness of macaque monkeys. Invest Ophthalmol Vis Sci 2000; 41: 1259-1269.

28 Troilo D, Nickla DL, Wildsoet CF. Choroidal thickness changes during altered eye growth and refractive state in a primate. Invest Ophthalmol Vis Sci 2000; 41: 1249-1258.

29 Fujiwara A, Shiragami C, Shirakata Y, Manabe S, Izumibata S, Shiraga F. Enhanced depthimaging spectral-domain optical coherence tomography of subfoveal choroidal thickness in normal Japanese eyes. Jpn J Ophthalmol 2012; 56: 230-235.

30 Moreno TA, O'Connell RV, Chiu SJ, Farsiu S, Cabrera MT, Maldonado RS et al. Choroid development and feasibility of choroidal imaging in the preterm and term infants utilizing SD-OCT. Invest Ophthalmol Vis Sci 2013; 54: 4140-4147.

31 Tan CS, Ouyang Y, Ruiz H, Sadda SR. Diurnal variation of choroidal thickness in normal healthy subjects measured by spectral domain optical coherence tomography. Invest Ophthalmol Vis Sci 2012; 53: 261-266.

32 Usui S, Ikuno Y, Akiba M et al. Circadian changes in subfoveal choroidal thickness and the relationship with circulatory factors in healthy subjects. Invest Ophthalmol Vis Sci 2012; 53: 2300-2307. 